for supplying the chicks, and the staff in the five public health laboratories for their cooperation.

1 Kurtz JB, Mortimer PP, Morgan-Capner P, Shafi MS, White GBB. Rubella antibody measured by radial haemolysis. Characterisation and performance of a simple screening method for use in diagnostic laboratories. fournal of Hygiene 1980;84:213-2.

2 Mortimer PP. Mumps prophylaxis in the light of a new test for antibody BrMed $\mathcal{F}$ 1978;ii:1523-4.

3 Gershon AA, Krugman S. Measles virus: In: Lennette EH, Schmidt NJ, eds. Diagnostic procedures for viral, rickettsial and chlamydial infections. 5th ed. Washington: American Public Health Association, 1979:665-93.
4 Department of Health and Social Security. Statistics and research. London: DHSS, 1987. (SR2B.)

5 Office of Population Censuses and Surveys. Communicable disease statistics. London: OPCS, 1987. (MB2.)

Centers for Disease Control. Measles in United States, first 26 weeks 1987. MMWR 1988;37:53-7.

7 Rabo E, Taranger J. Scandinavian model for eliminating measles, mumps, and rubella. Br Med $\mathcal{F}$ 1988;289:1402-4.

8 Nokes DJ, Anderson RM, Anderson MJ. Rubella epidemiology in south east England. Foumal of Hygiene 1986;96:291-304.

(Accepted 6 September 1988 )

\section{Sclerosis of lytic bone metastases after disodium aminohydroxypropylidene bisphosphonate (APD) in patients with breast carcinoma}

\author{
A R Morton, J A Cantrill, G V Pillai, \\ A McMahon, D C Anderson, A Howell
}

University Departments of Medicine and Pharmacy, Hope Hospital, Salford M6 8HD

A R Morton, MRCP, research fellow

J A Cantrill, MPS, staff pharmacist

G V Pillai, MPS, pharmacist

DC Anderson, FRCP,

professor of endocrinology

\section{Department of Medical} Oncology, Christie

Hospital and Holt Radium

Institute, Manchester

A McMahon, BSC, research

nurse

A Howell, FRCP, senior

lecturer in medical oncology

Correspondence to:

Dr Howell.
Bisphosphonates, which are potent inhibitors of osteoclast function, reduce morbidity from bone metastases when used with systemic chemotherapy. ${ }^{1}$ The contribution of each treatment in any patient is not known. We assessed the effect of disodium aminohydroxypropylidene bisphosphonate (APD) used either alone or in patients receiving endocrine treatment whose disease was progressing.

\section{Patients, methods, and results}

We studied 16 patients with breast carcinoma who had progressing metastases in bone for six months or until their disease progressed again despite treatment. APD (Ciba-Geigy) was administered intravenously (30 $\mathrm{mg}$ in $500 \mathrm{ml}$ of $0.9 \%$ saline over two hours) weekly for four weeks and thereafter fortnightly. At each visit we measured serum calcium, albumin, and phosphate concentrations; alkaline phosphatase activity; and ratios of fasting urinary calcium to creatinine and hydroxyproline to creatinine concentrations. Serum concentrations of osteocalcin and tumour markers (carcinoembryonic antigen and carbohydrate antigen $15: 3^{2}$ ) were measured monthly. Pain was scored on a linear analogue scale and the Karnofsky performance state assessed at each visit. A baseline isotope bone scan and plain radiographs of the chest, thoracolumbar spine, and pelvis were obtained. Radiography was repeated every six weeks. Evidence of sclerosis in a previously lytic lesion in the absence of new lesions was taken as a response to treatment. Statistical analysis was by Friedman's non-parametric two way analysis of variance.

Patients tolerated the infusions of APD well. Eight patients were withdrawn within six months with evidence of progressing disease (three in bone, five at other sites).

Effect on symptoms-Patients' perception of their pain, expressed as a percentage on the linear analogue scale, decreased significantly $(p<0.01)$ from a median of $69 \%$ (range $42-90 \%$ ) to $32 \%(5-75 \%)$ but was unrelated to radiological response. The Karnofsky performance state improved from a median of 70 (range 50-80) to $80(60-90)(\mathrm{p}<0.025)$.

Effects on biochemical variables-No significant change in serum calcium or phosphate concentrations occurred. The molar ratio of urinary calcium to creatinine concentrations fell significantly from a median of 0.63 (range $0 \cdot 10-1 \cdot 38)$ to $0.12(0.02-0.67)$ $(p<0.001)$ after the first infusion of APD. Patients who showed radiological improvement tended to have particularly low urinary ratios. The ratio rose dramatically in three patients whose disease progressed in bone. No significant change in alkaline phosphatase activity, osteocalcin concentration, or ratio of urinary hydroxyproline to creatinine concentrations was seen.

Antitumour effect-At six months radiological evidence of sclerosis of lytic metastases (figure) was seen in four patients and no change in the other four.
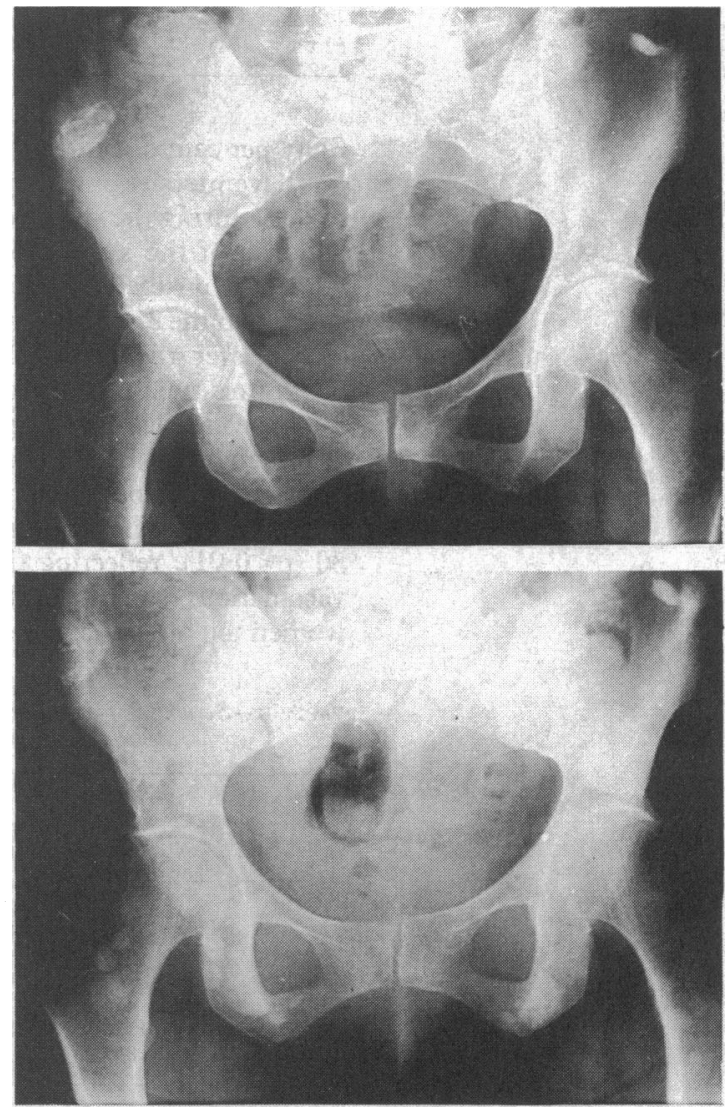

Lytic bone metastases before (top) and after (bottom) treatment with $A P D$

Concentrations of both carcinoembryonic antigen and carbohydrate antigen 15:3 fell in three patients (two who had a partial response and one whose disease remained stable), remained unchanged in eight patients, and rose in five.

\section{Comment}

APD produced sclerosis of lytic metastases in four patients, and in four others the disease remained stable by radiological criteria over six months. Patients who responded to APD seemed to show greatest suppression of bone resorption as reflected in their low ratios of urinary calcium to creatinine concentrations. Tumour marker concentrations fell in three of the 
eight patients who had a stable or partial response, suggesting that, in addition to causing sclerosis of bone, APD may have an antitumour effect. Factors released from tumours stimulate osteoblastic activity ${ }^{3}$ and osteolysis mediated by osteoclasts. ${ }^{4}$ APD is a potent inhibitor of osteoclast function, and such inhibition combined with the osteoblastic factors produced by tumour cells may explain the bony sclerosis seen in this study.

APD reduces morbidity from lytic metastases when used in conjunction with standard chemotherapy, ${ }^{1}$ and this study shows that it may be active when used alone.

We are grateful to the staff of the day ward, Christie
Hospital; the departments of chemical pathology at Christie Hospital and Withington Hospital; Mrs $\mathrm{H}$ Morten and $\mathrm{Mr}$ $\mathrm{J}$ Turnbull for performing the radioimmunoassays; and Ciba-Geigy Ltd for supplies of APD and for financial support.

1 Van Holten-Verzantvoort ATh, Bijvoet OLM, Hermans J, et al. Reduced morbidity from skeletal metastases in breast cancer patients during long-term bisphosphonate (APD) therapy. Lancet 1987;ii:983-5.

2 Pons-Anicet DMF, Krebs BP, Mira R, Namer M. Value of CA 15:3 in the follow-up of breast cancer patients. Br f Cancer 1987;55:567-9.

Pfeilschifter J, D'Souza SM, Mundy GR. Effects of transforming growth factor- $\beta$ on osteoblastic osteosarcoma cells. Endocrinology 1987:121:212-8.

Ibbotson KJ, Harrod J, Gowen $M$, al Human recombint grow vitro. Proc Natl Acad Sci USA 1986;83:2228-32.

(Accepted 8fuly 1988)

\section{Tolerance of intravenous indomethacin treatment for premature infants with patent ductus arteriosus}

\author{
Margaret Walters
}

Merck Sharp and Dohme Ltd, Hoddesdon, Hertfordshire EN11 9BU Margaret Walters, BSC, adverse experiences monitor
Following the observation that prostaglandin $E_{1}$, dilates the ductus arteriosus in fetal and neonatal animals, indomethacin-a potent inhibitor of prostaglandin synthesis- has been used for some years as an alternative to surgical ligation of the patent ductus arteriosus in premature infants with cardiac failure.

Giving the drug by mouth has been associated with variable absorption and local effects in the gastrointestinal tract. ${ }^{2}$ An intravenous formulation was developed to ensure bioavailability, and this report concerns the adverse event profile in 2482 premature infants who received intravenous indomethacin (Indocid PDA).

\section{Methods and results}

Since 1980, 2482 preterm neonates in the United Kingdom have received intravenous indomethacin (recommended dosage $0.2 \mathrm{mg} / \mathrm{kg}$ intravenously with second and third doses given at 12-24 hour intervals if required) on a named patient or humanitarian basis. All supplies were accompanied by a request to report any adverse events to Merck Sharp and Dohme forthwith (cases not resulting in a report were not followed up). Treatment was contraindicated in infants with proved or suspected infections; evidence of necrotising enterocolitis; gastrointestinal bleeding; intraventricular haemorrhage; coagulation defects; and appreciable impairment of renal function.

Reports of adverse events in 146 neonates $(6 \%)$ were reviewed; of these, 73 (3\% of total) were rated as being possibly, probably, or definitely related to the drug treatment by the reporting physician. The table gives details of the adverse events reported. None of the 61 deaths reported were considered by the reporting physicians to be drug related. In three cases in which infants had other life threatening conditions from birth the effect of indomethacin on renal function might have contributed to the death. The cases of renal impairment observed were not usually sustained or serious, but in cases of pre-existing renal impairment (when indomethacin is contraindicated) treatment may provoke severe deterioration. Increased creatinine concentrations and hyponatraemia were reported, but both these and renal function returned to normal on closure of the patent ductus arteriosus or cessation of drug treatment. The reported instances of gastro- intestinal haemorrhage were generally detected by nasogastric aspiration two to three days after administration of indomethacin, and most resolved within the next few days with no reported complications.

Details of adverse events occurring in 146 out of 2482 infants treated with intravenous indomethacin for patent ductus arteriosus and of those related to drug treatment in 73 infants ${ }^{\star}$

\begin{tabular}{|c|c|c|c|c|c|}
\hline \multirow[b]{2}{*}{ Adverse event } & \multirow{2}{*}{$\begin{array}{l}\text { Total } \\
\text { No of } \\
\text { reports }\end{array}$} & \multirow{2}{*}{$\begin{array}{c}\text { No } \\
\text { related } \\
\text { to drugt }\end{array}$} & \multicolumn{3}{|c|}{ Severity } \\
\hline & & & Mild & Moderate & Severe \\
\hline \multicolumn{6}{|l|}{ Intraventricular } \\
\hline Gastrointestinal bleeding & 19 & $18(0.73)$ & 8 & 5 & 5 \\
\hline Necrotising entercolitis & 24 & $13(0.52)$ & 2 & 4 & 7 \\
\hline Renal failure or shutdown & 15 & $10(0.4)$ & $i$ & 5 & 4 \\
\hline Fall in urea concentration & 12 & $12(0.48)$ & 4 & 6 & 2 \\
\hline Hyponatraemia & 18 & $16(0.65)$ & 2 & 10 & 4 \\
\hline \multicolumn{6}{|l|}{ Increased creatinine } \\
\hline concentration & 6 & $5(0 \cdot 2)$ & 0 & 5 & 0 \\
\hline Decreased platelet function & 2 & $2(0.08)$ & 2 & 0 & 0 \\
\hline Death & 61 & 0 & & & \\
\hline Seizures & 2 & 0 & & & \\
\hline Jaundice & 8 & 0 & & & \\
\hline
\end{tabular}

*Total numbers of events exceed the numbers of infants affected. † As determined by reporting physician. Values in parentheses are percentages of 2482 infants.

\section{Comment}

The rate of successful closure of a patent ductus arteriosus with oral indomethacin has been highly variable $(18-85 \%)^{3}$ but appears to be less variable with intravenous treatment $(79-91 \%){ }^{2}$ The best responses are achieved when neonates are treated in the first two weeks of life.

A review of indomethacin in the treatment of patent ductus arteriosus lists as the most important adverse effects transient impairment of renal function and decreased platelet function with possible clinical bleeding, including gastrointestinal and intraventricular haemorrhage ${ }^{4}$ Our findings in a large series of patients identified changes in renal function with associated electrolyte imbalance and gastrointestinal bleeding as the most common drug related effects. Necrotising enterocolitis and intraventricular haemorrhage were also prominent, although the haemorrhage was not often considered by the physician to be related to treatment. The relation between drug administration and necrotising enterocolitis and intraventricular haemorrhage is difficult to assess as preterm infants with patent ductus arteriosus are predisposed to these conditions, and studies have failed to detect an enhanced risk with indomethacin treatment. ${ }^{5}$

In conclusion, these data on 2482 preterm infants treated with intravenous indomethacin indicate that the incidence of adverse events is low. The commonest unwanted effects were transient impairment of renal function and gastrointestinal bleeding, which resolved on withdrawal of the drug. The efficacy of intravenous indomethacin in patent ductus arteriosus is well docu- 